\title{
The Effect of Passive Opium Smoking on Cardiovascular Indices of Rabbits with Normal and Ischemic Hearts
}

\author{
Siyavash Joukar ${ }^{1, *}$, Hamid Najafipour ${ }^{2}$, Reza Malekpour-Afshar ${ }^{3}$, Fatemeh Mirzaeipour ${ }^{4}$ and \\ Hamid Reza Nasri ${ }^{4}$ \\ ${ }^{I}$ Department of Physiology and Physiology Research Center \\ ${ }^{2}$ Department of Physiology, Physiology Research Center and neuroscience Research Center \\ ${ }^{3}$ Department of Pathology and neuroscience Research Center \\ ${ }^{4}$ Department of Cardiology and Physiology Research center; Kerman University of Medical Sciences, Kerman, Iran
}

\begin{abstract}
Some Asian people believe that opium can protect the cardiovascular system. To assess this belief, we investigated the effect of passive opium smoking (POS) on cardiovascular indices in rabbits with ischemic and non-ischemic hearts.

Rabbits ( $=43$ ) were divided into control (CTL), short term opium (SO) and long term opium (LO) groups. SO and LO groups were exposed to opium smoking for 3 days and 4 weeks, respectively. ECG, blood pressure (BP), left ventricular pressure and cardiac troponin I levels were recorded. Isoproterenol (ISO) was injected to induce cardiac ischemia and after $4 \mathrm{~h}$ the above variables were measured along with cardiac histopathology assessment.
\end{abstract}

All groups showed significant increments in troponin I level $(\mathrm{P}<0.05)$ except the CTL group. This trend was more obvious in ISO-treated groups. Mean arterial pressure (MAP) significantly decreased in all groups $(\mathrm{p}<0.05)$ except the LO group. Opium exposure attenuated ISO-induced myodegeneration but augmented tissue congestion and hemorrhage.

In conclusion, higher troponin I serum level and ECG changes were found in passive opium smoking groups. This evidence is against the belief that opium can protect the cardiovascular system.

Keywords: Opium smoking, cardiac ischemia, cardiac troponin I, histopathology.

\section{INTRODUCTION}

Opium is the air-dried milky exudate obtained by incising the unripe capsules of Papaver somniferum L., one of the oldest medicinal plants. More than half of the world's opiate users (7.8 million) are Asian with the highest prevalence rates reported from Iran, Kyrgyzstan and Laos [1]. Easy availability and a traditional belief about the beneficial effects of opium on cardiovascular disease (CVD) increase the prevalence of its consumption in these societies. Available human studies are limited on clinical and epidemiological grounds as well as the presence of confounding factors. Differences in constituents, dose, route and frequency of opium consumption and association with other substances (tobacco and cigarette smoking, alcohol and narcotic or tranquilizer drugs) are examples of confounding factors. Most studies indicated deleterious effects of opium on the cardiovascular system [2-4] and some did not find a relationship between opium and CVD $[5,6]$. In addition, some studies have shown

*Address correspondence to this author at the Department of Physiology and Physiology Research Center, Kerman University of Medical Sciences, Medical Faculty, Bulvd. 22 Bahman, Postal Code 7616914111, Kerman, Iran; Tel: +98 341 3220081; Fax: +98 341 3220081;

E-mails: Jokar@kmu.ac.ir, sjokar@gmail.com that morphine, one of the components of opium, has cardioprotective functions [7].

In present study we assessed the effects of short and long term opium smoking (most current consumption form in Iran) in a controlled animal model. Cardiovascular indices such as ventricular function, blood pressure (BP), ECG and myocardial histopathology in rabbits with or without ischemic hearts were recorded.

\section{MATERIAL AND METHODS}

Experiments conformed to national guidelines for conducting animal studies (Ethic committee permission No 86/123KA - Kerman University of Medical Sciences) and performed on 43 New Zealand White rabbits weighing between 2 and $3.5 \mathrm{~kg}$ (purchased from Pasteur institute of Iran). Animals divided to control (CTL), short-term opium (SO) and long term opium (LO) groups. Each group contains two subgroups, animals with normal or ischemic hearts.

\section{Apparatus for Passive Smoking}

The apparatus for opium smoking was made in the Physiology Research Center of Kerman University (Iran). This equipment includes a circular plate with several succes- 
sive rows of holes with equal distance for opium loading. The apparatus is programmed to rotate the plate such that 4 opium pieces (250 mg each) are released every $6 \mathrm{~h}$ in a vessel over a heater placed under the apparatus. The heater was programmed to be switched on a few seconds before opium releasing and continue for $5 \mathrm{~min}$ to convert the opium pieces to vapor/smoke and release towards the animal cage. The apparatus and heater were in a small room with $2 \times 1.5 \times 1.5 \mathrm{~m}$ dimensions containing an animal cage and connected by a $7.6 \mathrm{~cm}$ diameter tube to the outside of the animal room. Every $20 \mathrm{~min}$ after vapor/smoke production, a programmed automatic fan ventilates smoke and replaces room air. Animals were under this environment for short term (3 days) or long-term (4 weeks) periods. A 4 week period is sufficient to establish dependency in rabbits.

To confirm dependency in the long-term opium group, naloxone $(4 \mathrm{mg} / \mathrm{kg}$ intraperitoneally) was injected to some animals randomly. They showed withdrawal signs including marked grooming, head twitch, increased motility, mydriasis, ptosis, and writhing as previously described [8].

\section{Surgical Preparation and Experimental Protocol}

Animals were anaesthetized initially by injection of sodium thiopental (50 mg/kg intraperitoneally) and thereafter maintained with $1 \%$ halothane in a $30 \% \mathrm{O}_{2}-69 \% \mathrm{~N}_{2} \mathrm{O}$ mixture during the surgical procedure. On completion of surgery, the gaseous anesthesia was discontinued and animals were kept anaesthetized with sodium thiopental $(10 \mathrm{mg} / \mathrm{kg} / \mathrm{h}$, intraperitoneally) throughout the course of the experiment. The trachea was cannulated, with animals breathing spontaneously throughout the experiment. Deep anesthesia was maintained throughout as judged by the absence of withdrawal response to a pinch stimulus applied to the hind limb. BP was recorded throughout the experiment through a heparinized saline filled $(7 \mathrm{u} / \mathrm{ml})$ cannula inserted into the left carotid artery and connected to a pressure transducer and Physiograph (Beckman R612, USA). Another cannula was inserted into the left ventricle through the right carotid artery for left ventricular pressure recording and calculation of Max $\mathrm{dp} / \mathrm{dt}$, the maximal rate of change in left ventricular pressure with time, an index of myocardial contractility. An ear vein line was used for blood sampling. At the end of the surgical procedure, the animal was left for $1 \mathrm{~h}$ to minimize surgical stress. Thereafter baseline ECG and BP were recorded and a blood sample was taken for determination of plasma cardiac troponin I levels (a biomarker of cardiac muscle injury). Then isoproterenol (ISO) $3 \mathrm{mg} / \mathrm{kg}$ intraperitoneally was injected to induce myocardial ischemia in ischemic subgroups $[9,10]$. During the following $4 \mathrm{~h}$, vital signs were monitored continuously. At the end of $4 \mathrm{~h}$ after BP and left ventricular pressure record, blood sample was taken again for final troponin I measurement. After centrifugation of the blood, the serum was separated and stored at $-20^{\circ}$ for maximum of 2 weeks until troponin I measurement by VIDAS troponin I ultra assay, which is an enzyme-linked fluorescent immunoassay performed using an automated VIDAS instrument.

\section{Electrocardiography}

ECG electrodes were connected to the animal limbs and chest after shaving the related areas and ECGs were recorded using an Electrocardiograph (Yamaguchi- YM-102, Japan). The baseline recordings were made after animal was stabilised from surgical stress i.e. at the start of the experiment and the final recordings were made at the end of $4^{\text {th }} \mathrm{h}$ of the experiment. Then the ECGs were evaluated by 2 cardiologists (F. M. and HR. N.) which were blinded of animal group's names. Alterations, such as ST-segment elevation or depression and $\mathrm{Q}$ wave, were recorded as presence or absence in each lead.

\section{Histopathology}

After killing the animals, hearts were excised, rinsed with $0.9 \%$ saline and fixed with $10 \%$ buffered formalin. Each heart was cut into 4 segments from apex to bottom. Tissues were embedded in paraffin, and replicate $4 \mu \mathrm{m}$-thick sections were made at the basal, mid, and apical levels of the heart. Sections were stained with hematoxylin and eosin (H\&E), and examined microscopically by 2 pathologists (R. MA.) which blinded to animal groups. The slides were evaluated under a light microscope (Nikon, Tokyo, Japan) fitted with digital camera. Pathological indices including congestion, hemorrhage, necrosis, inflammatory cell infiltration and hypereosinophilic bundles were recorded. The observers examined at least 10 fields in each slide for Pathological indices and graded them for severity of changes using scores on a scale of severe $(++++)$, moderate $(+++)$, mild $(++)$, minimum $(+)$ and nil $(-)$.

ISO and sodium thiopental were purchased from Sigma (England). Opium was donated by the anti-drug section of Kerman Police (Iran). The alkaloids content of a sample of this opium was determined by gas chromatography and it included: morphine $16 \%$, codeine $18 \%$, thebaine $16 \%$, papaverine $13 \%$ and the other substances $(37 \%)$ of constituents.

\section{Data Analysis}

Values were expressed as the mean \pm SD. Mean arterial pressure ( MAP ), max dp/dt and troponin I, comparisons were performed between basal and $4^{\text {th }} \mathrm{h}$ values in each group by student paired t test and between different groups by oneway ANOVA followed by Tukey HSD (Honestly Significant Differences) multiple comparison post hoc test. Differences in the ECG variables were determined by Fisher's exact test. Histopathological changes of heart tissue were presented as mentioned in histopathology section.

\section{RESULTS}

\section{Arterial Blood Pressure}

The MAP at the $4^{\text {th }} \mathrm{h}$ decreased significantly $(\mathrm{p}<0.05)$ compared with basal level in all groups except the long term opium group. Comparison of MAP showed no significant difference among groups both at baseline and $4^{\text {th }} \mathrm{h}$ (Fig. 1).

\section{Maximum of $\mathbf{d p} / \mathrm{dt}$}

Changes in Max dp/dt during $4 \mathrm{~h}$ were not significant between groups. However, the trend of change was different in long opium group (LO) treated with ISO (Fig. 2). In this group no reduction in Max dp/dt was observed. 


\section{Plasma Cardiac Troponin I}

Plasma cardiac troponin I level was increased significantly in all groups except the control group during the $4 \mathrm{~h}$ experiment. Opium along with ISO-treated groups showed higher increment but not significant in troponin I level compared with other groups (Fig. 3).

\section{Electrocardiographic Data}

The animals number with ST segment elevation in ECG were high in ISO and LO +ISO groups compared with related controls (Table 1), but this differences was not significant. An ECG trace of 1 animal in the LO +ISO group is presented in Fig. (4).

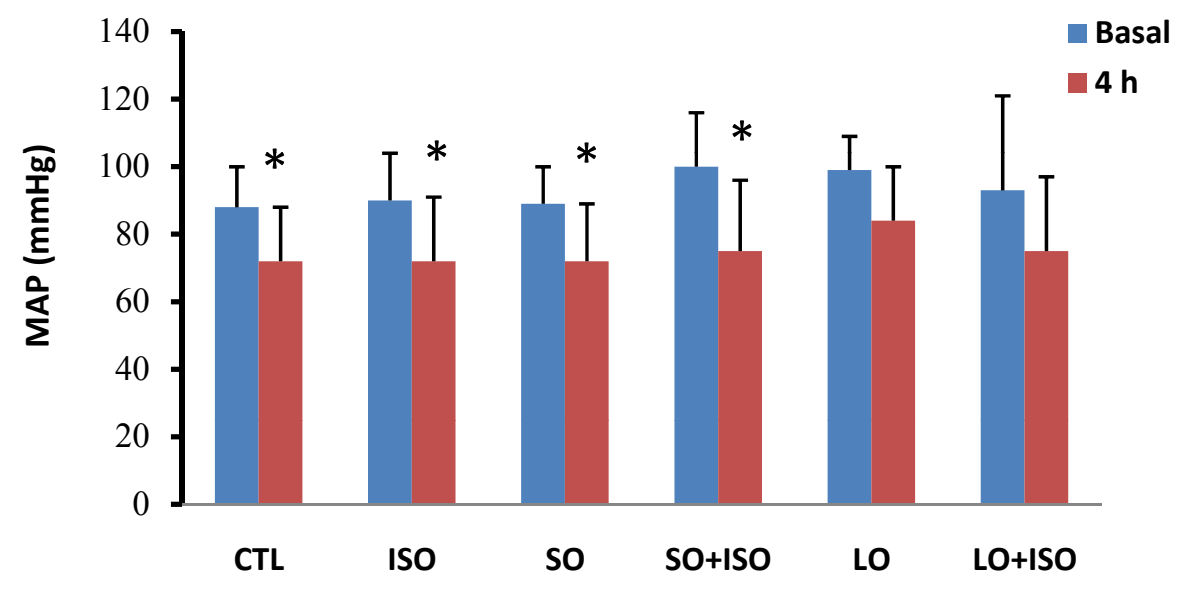

Fig. (1). Basal and $4^{\text {th }} \mathrm{h}$ of mean arterial pressure (MAP) in different animal groups. Values are means \pm SD. $n=6-8$; CTL, control; SO, short opium; LO, long opium; ISO, isoproterenol. * $\mathrm{P}<0.05$ compared with relative basal level.
CTL 150
so ${ }^{\mathrm{SO}_{\mathrm{N}} \mathrm{s}_{\mathrm{O}}}$
$10^{10}+150$

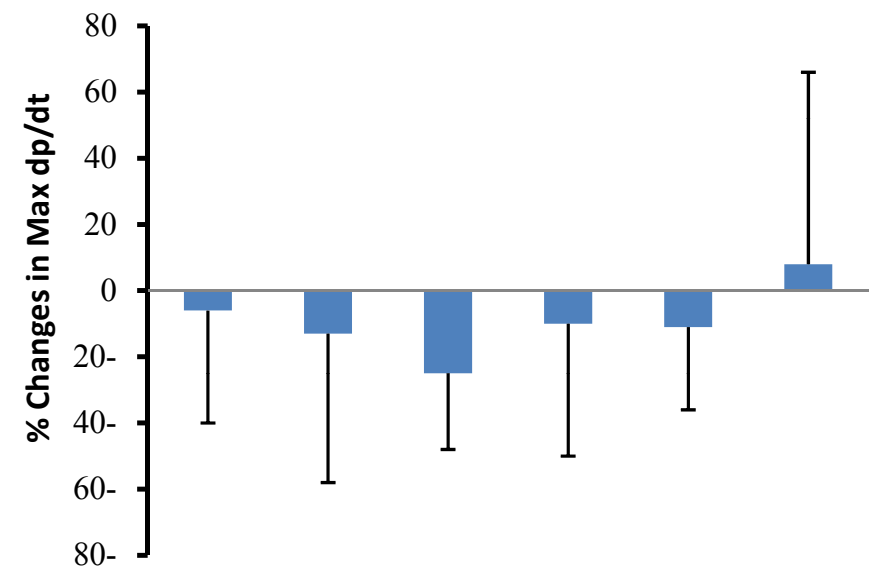

Fig. (2). Changes in Max dp/dt from basal levels during $4 \mathrm{~h}$ of experiment in different animal groups. Values are means $\pm \mathrm{SD}$. $\mathrm{n}=6-8$; $\mathrm{CTL}$, control; SO, short opium; LO, long opium; ISO, isoproterenol.

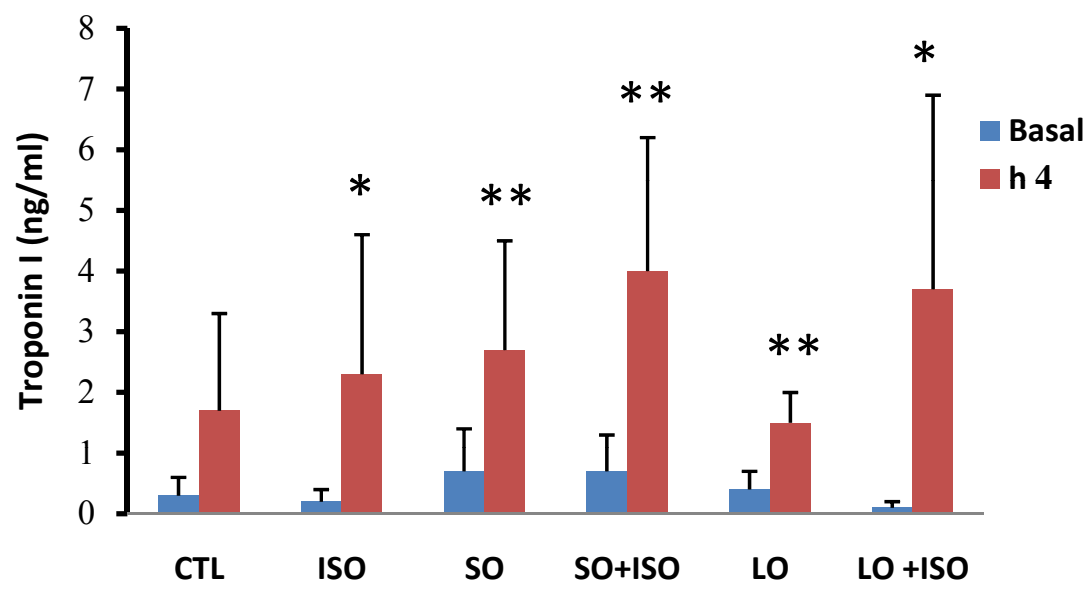

Fig. (3). Basal and 4th h plasma cardiac Troponin I levels in animal groups. Values are means \pm SD. $n=6-8$; CTL, control; SO, short opium; LO, long opium; ISO, isoproterenol. $* \mathrm{P}<0.05$ and ${ }^{* *} \mathrm{P}<0.01$ compared with relative basal level. 
Table 1. ECG Changes in Different Animal Groups

\begin{tabular}{|c|c|c|c|}
\hline Group & ST Depression (\% of Animals) & ST Elevation (\% of Animals) & Q Wave (\% of Animals) \\
\hline \hline CTL $(n=8)$ & 0 & 0 & 0 \\
\hline ISO $(n=8)$ & 28.6 & 42.9 & 0 \\
\hline SO (n=7) & 0 & 28.6 & 0 \\
\hline SO+ISO (n=8) & 0 & 25 & 0 \\
\hline LO (n=6) & 16.7 & 50 & 16.7 \\
\hline LO+ISO $(n=6)$ & 16.7 & 0 & 0 \\
\hline
\end{tabular}

n, number of animals; CTL, control; SO, short opium; LO, long opium; ISO, isoproterenol.
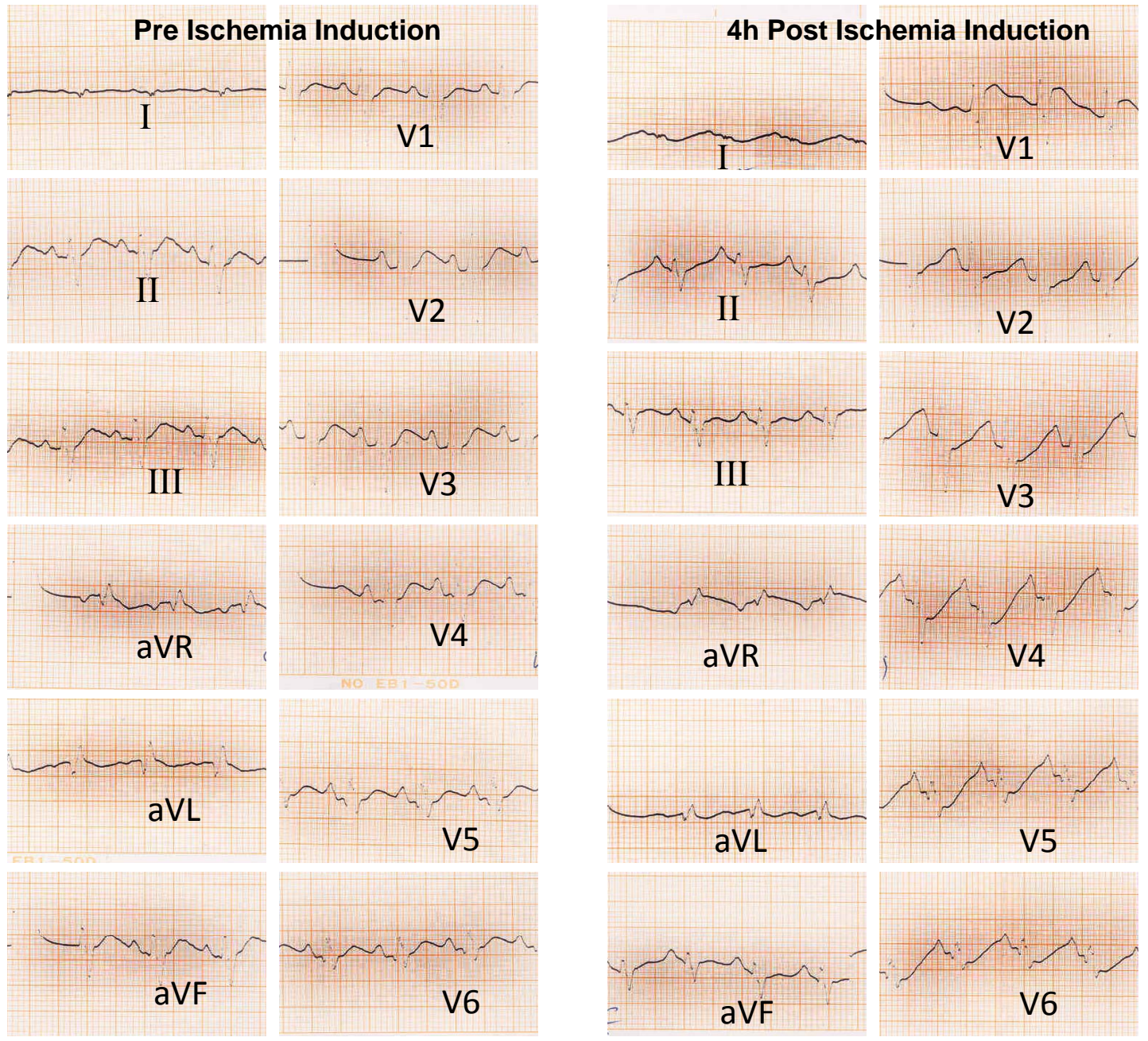

Fig. (4). Pre and 4 hours post ischemic induction ECG trace of 1 animal from the LO+ISO group that shows ST segment elevation in VI and aVR leads and ST segment depression in V2 $\rightarrow \mathrm{V} 6$ leads $4 \mathrm{~h}$ after ischemia induction.

\section{Histological Findings}

The heart tissue sections of control group did not show remarkable alterations. The ISO group showed mild to moderate hypereosinophilic bundles and subendocardial necrosis, moderate hemorrhage and mild leucocytes infiltration in heart tissue. Moderate tissue congestion and even bleeding was the prominent manifestation of the hearts tissue exposed to opium. Opium groups treated with ISO showed intensifi- cation in the hypereosinophilic bundles and leucocytes infiltration but without myocardial necrosis (Table 2 and Fig. 5).

\section{DISCUSSION}

The goal of this study was to determine the effects of opium smoking on cardiovascular parameters. The results indicated that opium smoking increased serum troponin I 
Table 2. Histopathological Assessment in Different Groups

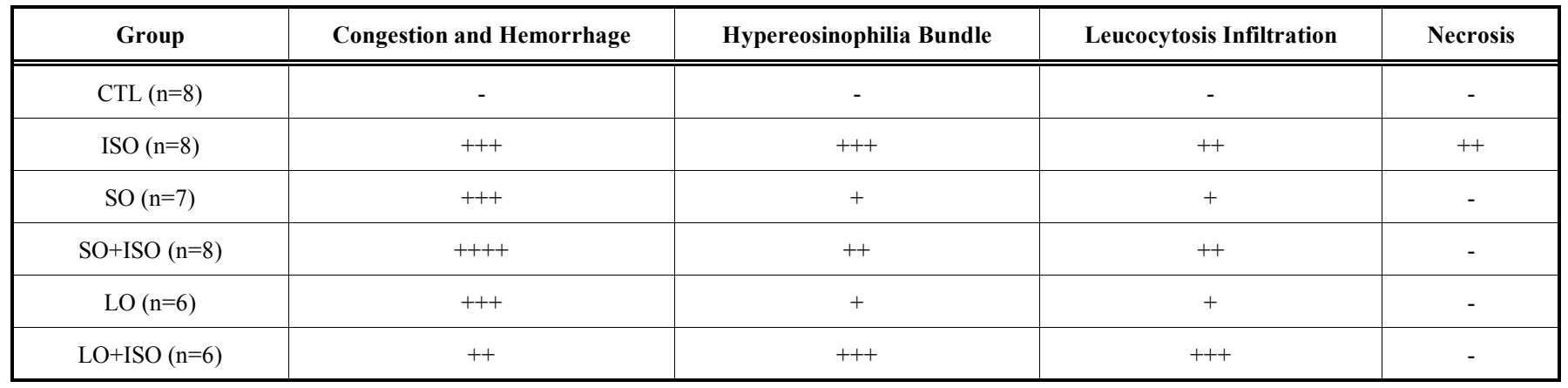

n, number of animals; CTL, control; SO, short opium; LO, long opium; ISO, isoproterenol; (++++), severe; (+++), moderate; (++), mild; (+), minimum; (-), nil.
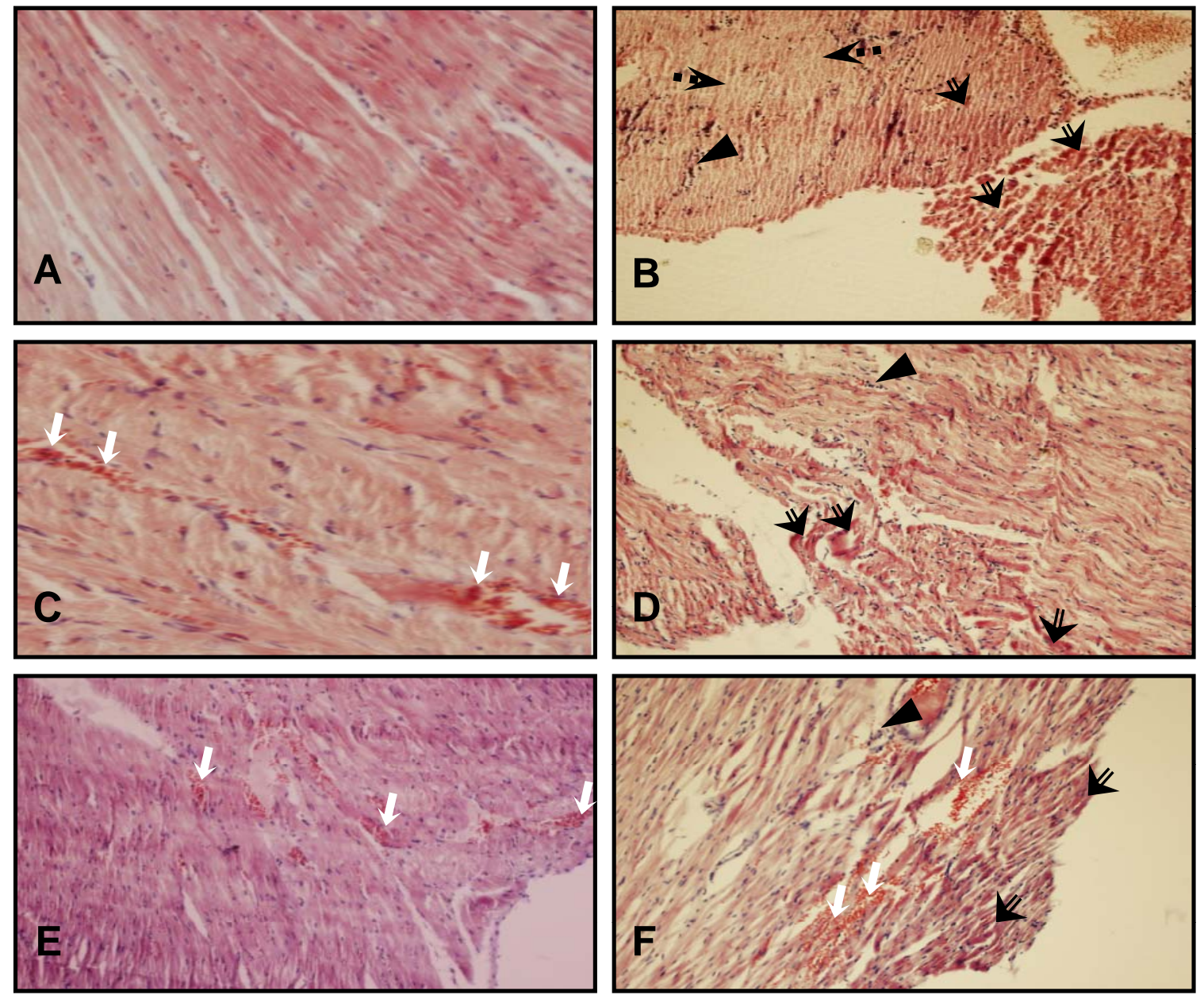

Fig. (5). H \& E stained sections of heart tissue. (A) CTL group heart section showing normal appearance of myocardial fibers. (B) Degeneration of myocardial fibers and leucocyte infiltration in a heart of ISO group. (C) Congestion of cardiac tissue in a heart of SO group. (D) Hypereosinophilic bundles and leucocyte infiltration in a heart of SO + ISO group. (E) Congestion and hemorrhage of cardiac tissue in a heart of LO group. (F) Hypereosinophilic bundles, leucocyte infiltration, congestion and hemorrhage of cardiac tissue in a heart of LO +ISO group. White arrow: congestion and hemorrhage, triangle: leucocyte infiltration, black arrow with empty tail: Hypereosinophilic bundles, black arrow with dotted tail: Necrosis. The magnification of $\mathrm{A}$ and $\mathrm{C}$ is $\times 400$ and others are $\times 100$.

level and aggravated ECG changes in animals with or without ischemic hearts. Intraperitoneal injection of ISO caused increased serum cardiac troponin I level, myocardial damage and ECG changes that is consistent with previous studies [9, $10]$.
Both short and long opium exposure in both ischemic and non-ischemic hearts significantly increased the serum cardiac troponin I levels compared to related basal levels. Bafghi et al. showed that mortality rate significantly increased in opium addicts hospitalized due to acute myocardial infarc- 
tion (MI) compared with non-addicts [3]. In a retrospective analysis, Peacock et al. reported that morphine therapy in acute decompensated heart failure (ADHF) raised serum troponin I levels significantly and increased adverse events including a greater frequency of mechanical ventilation, more ICU admissions, prolonged hospitalization and higher mortality [11]. Although, opium is a compound material that in addition to morphine includes other alkaloids and nonalkaloids substances, its effect on cardiac troponin release in our experiment were similar to the effect of morphine reported by Peacock et al. Overall, it seems that opium consumption potentiates cardiac stress during cardiac ischemia. Furthermore, in ischemic conditions, long term opium smoking not only did not recover ECG changes but also increased the number of the animals with ST segment elevation. This means that from an electrical activity point of view, opium smokers are probably more susceptible to heart ischemia when they consume opium to reduce their chest pain or blood pressure. In agreement with our study, Wallner et al. reported that ECG abnormalities are frequent in opiate addicts [12]. On the other hand, MAP decreased significantly in the presence or absence of ISO and short term opium during our experiment. The significant reduction of MAP during cardiac stress (surgical or ischemia) is a harmful effect in conditions where the heart needs a higher perfusion pressure. This phenomenon was attenuated in long term opium smoking groups, as it modulated the reduction of BP during experiments in ischemic conditions. Therefore, a protective like effect against ischemia seems to come into play with long term exposure to opium. The mild but non significant increase in Max dp/dt (Fig. 2) may be suggested as the possible mechanisms for this role. Previous studies have demonstrated that some opioid receptors ( $\mu$-type) mediate a pressor response $[13,14]$, while other receptors ( $\kappa$-type) mediate a depressor response [13]. Fontana et al. demonstrated that pressor effects of opioid peptides mediated by the autonomic nervous system during stress-induced acute episodes of BP increase in hypertensive patients [15]. In the present study, 1 possible explanation for BP stabilizing in long term opium consumption ischemic heart animals may be a greater $\mu$ opioid-mediated responsiveness with a greater mediated autonomic tone in the presence of long term opium using and stress.

Less degeneration but considerable congestion/hemorrhage was the most frequent histopathological findings in animal hearts with opium consumption. In a human study opiate addicted females presented lower platelet counts [16]. This may be a reason for congestion and hemorrhage in opium animal groups in our study. Less tissue damage in long term opium exposed animals in ischemic condition would be consistent with the study of Peart et al. that demonstrated morphine has a cardioprotective function [7]. Less drop in BP in the LO +ISO group may have caused delay in degeneration of myocardial cells during ischemia.

In conclusion, more troponin I serum level and ECG changes found in long term opium smoking groups are in contrast to the belief that opium may have preventive or ameliorating effect on CVD. However, opium may delay the myocardial degeneration process after ischemia induction. Further investigation is required to assess the underlying mechanism of opium smoke on heart function.

\section{ACKNOWLEDGEMENTS}

Financial support of the project by Kerman University of Medical Sciences and Health Services of Iran is acknowledged. We are grateful to the anti-drug section of Kerman Police (Iran) for supplying the opium.

\section{REFERENCES}

[1] Nissaramanesh B, Trace M, Roberts M. The rise of harm reduction in the islamic republic of Iran. The Beckley Foundation Drug Policy Programme July, 2005.

[2] Asgary S, Sarrafzadegan N, Naderi GA, Rozbehani R. Effect of opium addiction on new and traditional cardiovascular risk factors: do duration of addiction and route of administration matter? Lipids Health Dis 2008; 7: 42.

[3] Sadr BSM, Rafiei M, Bahadorzadeh L, et al. Is opium addiction a risk factor for acute myocardial infarction? Acta Med Iran 2005; 43: 218-22.

[4] Sadeghian S, Darvish S, Davoodi G, Salarifar M, Mahmoodian M, Fallah N. The association of opium with coronary artery disease. Eur J Cardiovasc Prev Rehabil 2007; 14: 715-7.

[5] Davoodi G, Sadeghian S, Akhondzadeh S, Darvish S, Alidoosti M, Amirzadegan A. Comparison of specifications, short term outcome and prognosis of acute myocardial infarction in opium dependent patients and nondependents. J Tehran Heart Center 2006; 1: 43-7.

[6] Azimzade-Sarwar B, Gholamreza YG, Narooey S. A case-control study of effect of opium addiction on myocardial infarction. Am $\mathrm{J}$ Appl Sci 2005; 2: 1134-5.

[7] Peart JN, Gross ER, Gross GJ. Opioid-induced preconditioning: Recent advances and future perspectives (Review). Vasc Pharmacol 2005; 42:211-8.

[8] Yamatsu K, Takagi H. Electeroencephalographic studies on morphine dependence in rabbit. Jpn J Pharmacol 1966; 2: 225-7.

[9] Pinelli A, Trivulzio S, Tomasoni L, Brennab S, Bonacina E, Accinni R. Isoproterenol-induced myocardial infarction in rabbits. Protection by propranolol or labetalol: a proposed non-invasive procedure. Eur J Pharm Sci 2004; 23: 277-85.

[10] Pinelli A, Trivulzio S, Tomasoni L, et al. Myocardial infarction non-invasively induced in rabbits by administering isoproterenol and vasopressin: protective effects exerted by verapamil. Fundam Clin Pharmacol 2004; 18; 657-67.

[11] Peacock WF, Hollander JE, Diercks DB, Lopatin M, Fonarow G, Emerman CL. Morphine and outcomes in acute decompensated heart failure: an ADHERE analysis. Emerg Med J 2008; 25: 205-9.

[12] Wallner C, Stollberger C, Hlavin A, Finsterer J, Hager I, Hermann P. Electrocardiographic abnormalities in opiate addicts. Addiction 2008; 103:1987-93.

[13] Feuerstein G, Siren AL. The opioid peptides. A role in hypertension? Hypertension 1987; 9: 561-5.

[14] Hill-Pryor C, Lindsey D, Lapanowski K, Dunbar JC. The cardiovascular responses to mu opioid agonist and antagonist in conscious normal and obese rats. Peptides 2006; 27: 1520-6.

[15] Fontana F, Bernardi P, Spampinato S, Boschi S, De Iasio R, Grossi G. Pressor effects of endogenous opioid system during acute episodes of blood pressure increases in hypertensive patients. Hypertension 1997; 29:105-10.

[16] Verde MCM, Díaz-Flores JF, Sañudo RI, Rodríguez REM, Díaz Romero C. Haematologic parameters in opiate addicts. Nutr Hosp 2003; 18:358-65. 